\title{
Die künftige Entwicklung des Versicherungsmarktes aus der Sicht der Praxis
}

\author{
von Karl Kornis *
}

Die allgemeine Verschlechterung der technischen Ergebnisse der Versicherungswirtschaft praktisch aller Länder zeigt, da $\beta$ die Assekuranz entgegen einer in der Offentlichkeit verbreiteten Meinung sehr wohl von der gesamtwirtschaftlichen Krise beeinflußt wird. Die jüngste Entwicklung beweist, daß wir in Österreich nicht auf einer Insel der Seligen leben. Kein Land kann sich von der weltweiten Entwicklung abkoppeln. Ebenso wie in der Bankenwelt, die international verflochten ist, verhält es sich auch in der Versicherungswirtschaft. Dies vor allem durch den steigenden Bedarf nach internationaler Rückversicherung, wo es $\mathrm{zu}$ einer immer stärkeren globalen Interdependenz kommt.

Was geschah seit dem 2. Weltkrieg?

Die Weltwirtschaft begann sich allmählich zu erholen. Eine lebhafte industrielle Entwicklung setzte ein, es kam zu einer starken Motorisierung in der ganzen westlichen Welt.

Die Versicherungswirtschaft folgte und erlebte ebenfalls einen raschen Aufschwung. Die Zinssätze dieser Zeit waren niedrig, man war also gezwungen, technische Gewinne zu realisieren. Unvertretbaren Wettbewerb gab es zu dieser Zeit kaum.

Die für die Versicherer glückliche Situation währte bis zur Mitte der 50er Jahre. Ein Beispiel : Noch im Jahre 1954 erzielten die gesamten Schaden- und Unfallversicherer in den USA (Leben wird ausgeklammert) einen Gewinn von 956 Millionen Dollar. Davon stammten 506 Millionen Dollar aus dem technischen Ergebnis (underwriting-result) und nur 450 Millionen Dollar aus dem finanziellen Ergebnis (investment income). Ab Mitte der 50er Jahre änderte sich jedoch das Bild. In vielen Ländern begann sich die Lohn- und Preisspirale zu drehen.

Ständig steigende Kosten führten zu einer Verminderung der Gewinne in der Sachversicherung. Sparprogramme und strenge Wirtschaftlichkeitsüberlegungen der Unternehmen waren die notwendige Folge. Der Inflationsentwicklung folgend begannen die Zinssätze zu steigen, was sich bald in finanziell höheren Ergebnissen der Versicherungsgesellschaften niederschlug. Das Rechnen mit einem ständig wachsenden Ein-

* Generaldirektor, Vorsitzender des Vorstandes der Ersten Allgemeinen VersicherungsAktiengesellschaft, Wien. Referat gehalten am 18. November 1982, beim Symposium des Verbandes Ósterreichischer Versicherungsmakler, Wien. 
kommen aus den Kapitalerträgen bei Erstellung der Bilanzen — in den 50er Jahren noch als tödliche Sünde angesehen - wurde stillschweigend immer mehr zur Praxis. Man war bereit, niedere oder unter Umständen gar keine Gewinne aus dem versicherungstechnischen Geschäft hinzunehmen. Die 70er Jahre waren von der Energiekrise gekennzeichnet. Pessimisten sagten voraus, daß in Zukunft nichts mehr so sein werde wie zuvor. Sie wurden nicht immer ernst genommen. Seit dem Zusammenbruch des Weltwährungssystems von Bretton-Woods 1971/72 und im Gefolge des Jom-KippurKrieges 1973 sowie des bekannten "Ölschocks" begann die Weltwirtschaft eine Talfahrt. Kürzungen und Abstriche wurden überall notwendig. Die Arbeitslosenraten stiegen in den meisten Ländern katastrophal. Die weltweite Rezession beeinflußte natürlich auch die Versicherungswirtschaft. Auch sie mußte von den Tagen eines fast automatischen Wachstums Abschied nehmen und dies bei steigenden Kosten.

Als Ausweg aus dieser Situation wendete sich ein Teil der Industriestaaten verstärkt den Entwicklungsmärkten und den Ölstaaten zu. Die Versicherungswirtschaft folgte diesem Trend. Tochtergesellschaften wurden im Ausland errichtet, ausländische Gesellschaften übernommen. Das Ergebnis dieser Expansion war ein ungezügelter Wettbewerb auf den internationalen Versicherungs- und Rückversicherungsmärkten mit entsprechendem Druck auf die Prämien. stellen.

Man kann die Problematik, die ich kurz geschildert habe, in fünf Thesen dar-

\section{These}

Weltweit besteht heute eine enorme U'berkapazität auf den Versicherungsmärkten, insbesondere auf dem Rückversicherungssektor. Diese " overcapacity" wird oft treffend als " uniformed capacity" oder, noch schärfer, als " ignorant capacity" bezeichnet. Gemeint sind dabei Gesellschaften, die sich zum Ziel gesetzt haben, mit allen Mitteln in etablierte Märkte einzudringen. So offerieren sie, oft in völliger Fehleinschätzung der Risken, Prämien und Konditionen, die, langfristig gesehen, auf keinen Fall die Schäden und die Kosten abdecken können.

\section{These}

Die rein nominelle Betrachtung der Zinseinnahmen, die Nichtberücksichtigung der Inflation, führt zu einem gefährlichen Trugschluß. Bei Berücksichtigung der Inflation entpuppt sich nämlich, daß der Höhenflug der Zinsen ein Mythos ist. Wenn man die reale Verzinsung betrachtet, das heißt die Rendite vermindert um die Inflationsrate, haben wir ein ganz anderes Bild. Wenn wir die Jahre 1972 bis 1981 betrachten und in Österreich die Sekundärmarktrendite langfristiger staatlicher Schuldverschreibungen analysieren, dann sehen wir, daß ihre Zinsergebnisse nur um 2 bis $3 \%$ über der Inflationsrate lagen. In den meisten OECD-Staaten ergab sich für viele Jahre eine gleichbleibende Situation mit ca. 3 Prozent über der Inflationsrate. Das heißt, daß der Rest der Nominalverzinsung nur ein Scheingewinn ist.

In den Jahren 1972 bis 1976 war der Unterschied zwischen der Inflationsrate und der Sekundärmarktrendite langfristiger staatlicher Schuldverschreibungen nicht bedeutend. In den folgenden Jahren, von 1977 bis 1981, entwickelte sich jedoch eine größere 
Realgewinnsituation, allerdings nur bis maximal 4 Prozent im Jahre 1981. Nun, will ein Unternehmen seine Substanz nicht von der tückischen Krankheit der Inflation aushöhlen lassen, so muß es seine Reserven und letztlich auch seine Kapitalbasis zumindest im Ausmaß der Inflationsrate verstärken. Ich glaube, das ist eine sehr wichtige Aussage. Unter Berücksichtigung dieser Tatsache ist der Ernst der Situation zu erkennen. Die technischen Ergebnisse befinden sich seit 1978 auf einer kontinuierlichen Talfahrt. Sie werden durch die nominellen Vermögenserträge - eben wegen der Inflation nur scheinbar ausgeglichen und im Jahre 1982 ist noch kein Trend zur Besserung zu verzeichnen.

\section{These}

Erlauben Sie mir, einiges zum sogenannten "Cash-flow-underwriting" zu sagen. Dieser Ausdruck steht für eine Gesellschaftspolitik, die in erster Linie auf den Umsatz ausgerichtet ist, mit dem Ziel, möglichst viel Mittel zur Vermögensveranlagung zu erhalten. Als "Cash-Flow" wird in der Versicherungswirtschaft - anders als in der Industrie, wo auch ein Anlagevermögen vorhanden ist - jener Betrag verstanden, der für die Veranlagung zur Verfügung steht, also im wesentlichen die reine Differenz von Einnahmen und Ausgaben, ohne Berücksichtigung der Erhöhung der Reserven. Das "Cash-flow-underwriting" führt häufig dazu, daß leichtfertig schlechte Risiken und niedrige Prämien akzeptiert werden, um über gesteigerte finanzielle Gewinne schließlich zu einem positiven " bottom-line-profit", einem Gewinn unter dem Strich, zu gelangen.

Vor allem in den Vereinigten Staaten war es eine zeitlang sogar Usus, Berechnungen darüber anzustellen, wie hoch man den kombinierten Schaden-Kostensatz über $100 \%$ ansteigen lassen könne, um noch immer durch die finanziellen Ergebnisse positive Resultate zu erzielen. "Cash-flow-underwriting" bedeutet eine gefährliche Abkehr von der alten und bewährten Forderung, daß allem voran ein positives technisches Ergebnis erwirtschaftet werden muß. Das finanzielle Ergebnis hingegen darf nur dazu verwendet werden, um die inflationäre Aushöhlung der Reserven zu kompensieren und um die Eigenkapitalbasis für die Zukunft zu stärken, selbst wenn dies in manchen Ländern steuerlich stark belastet ist.

$\mathrm{Daß}$ heute die Versicherungswirtschaft in Österreich auf einer relativ soliden Basis steht, verdanken wir unseren Vorgängern. Unseren Vorgängern, die an die Zukunft glaubten, und die in diese Zukunft investierten. Warum sollten wir diejenigen sein, die damit aufhören?

Das Versicherungsgeschäft, auch das Rückversicherungsgeschäft, ist seiner Natur nach ein langfristiges Geschäft. Volumen-orientiertes Verhalten mit Inkaufnahme technischer Verluste kann sich auf die Dauer nur verhängnisvoll auswirken. Die Annahme, daß hohe Zinserträge diese Verluste ausgleichen könnten, mag im Einzelfall bei kurzfristiger Betrachtung zutreffen, ist aber bei langfristiger Analyse absolut unzulässig. Der "Cash-flow" kann, schneller als erwartet, versiegen oder sogar negativ werden, wenn sich die Schere zwischen wettbewerbsbedingt stagnierenden oder gar sinkenden Prämien und den nicht zuletzt aufgrund des dumping-Effektes des "Cashflow-underwriting" inflationsbedingt steigenden Schäden sowie Kosten weiter öffnet. Hiezu kommt noch das Risiko der Vermögensanlagen. Ein Underwriter, der sich auf 
die Wunderkräfte des "Cash-flow" verläßt, täuscht sich, denn er darf keineswegs davon ausgehen, daß die Zinserträge stets auf demselben Niveau bleiben oder gar noch steigen werden. Es ist daher die Aufgabe der Assekuranz, nur auf der Basis eines kaufmännisch vernünftig kalkulierten Preises Risiken zu übernehmen. Es kann nicht ihre Aufgabe sein, mit der Fieberkurve der Zinssätze zu spekulieren. Das hieße nämlich, daß neben der Deckung des eigentlichen Versicherungsrisikos auch auf der nicht technischen Seite erhebliche und unabsehbare Risiken eingegangen werden. Der Präsident des Gesamtverbandes der Deutschen Versicherungswirtschaft, Dr. Georg Büchner, hat dies sehr zutreffend als Verzicht auf Sicherheit bezeichnet. Beim "long-tail-business", Geschäften mit langer Regulierungsdauer, kommt dazu noch inflationsbedingt die Verteuerung der Schäden. In der Haftpflichtversicherung z.B. beinhaltet jede Prämienkalkulation neben der eigentlichen versicherungstechnischen Beurteilung zwangsläufig auch Schätzungen über das zukünftige Preisniveau, über die zukünftigen Haftpflichtbestimmungen, über die zukünftigen Maßstäbe für Sorgfalts- und Beweispflichten sowie über die zukünftigen Entschädigungsmaßstäbe. Man denke auch an Invaliditätsfälle, bei denen dynamisierte Rentenansprüche entstehen oder an die Produktehaftpflicht, bei der die Konsequenzen für die Geschäftswelt gar nicht abgeschätzt werden können.

Schließlich ist noch die technologische Entwicklung zu nennen, die zu immer größeren und komplizierteren Risiken führt. Denken Sie an Ölraffinerien, HochseeBohranlagen, Supertanker, noch größere Jumbo-Jets, Kernkraftwerke, Dammkonstruktionen, Telesatelliten usw.

Professor Eichhorn hat in einem im Jahre 1981 erschienenen Aufsatz - Energieverteuerung und ihre Auswirkung auf den Geldwert und die Versicherung - auf das Problem der Direkt- und Rückversicherer hingewiesen, und er meinte, daß das Wachstum ihrer Reserven wenigstens annährend mit dem Größenwachstum neuer Risiken Schritt halten sollte. Das heißt, während sich das Wachstum der Prämien und der Vermögensanlagen der Schaden- und Unfallversicherer in Deutschland im Vergleich zur Entwicklung der Baukosten solcher Groß-Objekte noch 1955 bis 1960 annähernd im Gleichschritt vollzog, öffnete sich die Schere in den folgenden Jahren zunehmend zu Lasten der Versicherer. Diese Ausführungen über die Probleme, die sich aus der Langfristigkeit des Versicherungsgeschäftes ergeben, machen deutlich, daß dieser Langfristigkeit der Verpflichtungen nicht mit der kurzfristigen - oder besser : kurzatmigen - Mentalität des "Cash-flow-underwriting" begegnet werden kann. Als anschauliches Beispiel zur Untermauerung dieser Aussage darf die jüngste Entwicklung in Kanada gelten. Dort hat sich 1982 das Geschäft so katastrophal entwickelt, daß bereits zwei große Gesellschaften, STRATHCONA und PITTS, in ernste Schwierigkeiten gerieten. Die Aufsichtsbehörde hat ihnen die Genehmigung zur Zeichnung von Neugeschäft entzogen und die Vermögensanlagen dieser Gesellschaften unter Kontrolle gestellt. Eine Schädigung der Versicherungsnehmer konnte gerade noch verhindert werden.

\section{These}

Technische Verluste werden nicht nur durch übersteigerten Wettbewerb hervorgerufen, sondern sind mitunter auch die Folge staatlicher Eingriffe. 
Ein klassisches Beispiel hiefür ist das Kfz-Haftpflichtversicherungsgeschäft. Wie Sie wissen, ist die Kfz-Haftpflicht-Prämie nicht nur in Österreich, sondern auch in vielen anderen Ländern Europas staatlich geregelt. Diese Regelung ist uiberall ein Problem. In einer Zeit des Höhenflugs der Zinsen fanden wir in diesem Bereich zwar noch einen gewissen Ausgleich, aber es ist doch ein Dauerproblem geblieben. Ich möchte damit nicht sagen, daß hier unbedingt eine Anderung erfolgen sollte, weil es uns nicht zusteht, gesetzliche Maßnahmen ändern zu wollen. $\mathrm{Zu}$ diesem Zeitpunkt wäre es nach meiner persönlichen Beurteilung bei der heutigen Marktsituation in Österreich auch nicht besonders wünschenswert. Wir haben hier in diesem Bereich ohnehin genügend große Probleme, die durch eine Freigabe der Kfz-Haftpflichtprämie nur noch akuter würden. Trotzdem habe ich es erwähnt, da wir sowieso mit den Prämienerhöhungen immer nachhinken und diese Entwicklung auf die Ergebnisse der Versicherer drückt.

\section{These}

Wenn man die Zeichen der Zeit richtig interpretiert, dann muß sich auch ein modernes Versicherungsmanagement auf die klassischen Forderungen der Aktuarwissenschaft zurückbesinnen, und zwar: Ein Versicherungsunternehmen muß über eine ausreichende Eigenkapitalbasis verfügen, um das versicherungstechnische Änderungsrisiko tragen zu können und um für unvorhersehbare Katastrophen gerüstet zu sein. Aus dieser Erkenntnis heraus gelten in der EG strenge Solvabilitätsbestimmungen, die die Eigenkapitalausstattung der Versicherungsunternehmungen regeln. Vereinfacht ausgedrückt bedeutet dies für die Sachversicherer die Notwendigkeit, über Eigenmittel in Höhe von etwa 16 Prozent der Prämie zu verfügen. Dazu gibt es zwei verschiedene Kalkulationsmethoden : die eine basiert auf der Prämie, die andere auf dem Schadenssatz. Diese Minimalforderung von 16 Prozent dürfte gerade ausreichen. Nach dieser internationalen " tour d'horizon" darf ich mich nun kurz auf die Situation in Österreich konzentrieren, wobei ich auch hier die Probleme der Lebensversicherung ausklammern möchte.

Wenn wir in Österreich das Verhältnis Prämien zu Leistungen vergleichen, ergibt sich auch bei uns ein ungünstiges Bild. Der flüchtige Betrachter könnte zur Auffassung gelangen, daß diese Entwicklung in Österreich noch nicht voll durchgeschlagen hat. Ich möchte hier folgende Parallelprozentsätze, auf Prämie und Leistung bezogen, bringen.

Von 1972 bis 1980 erhöhten sich die Prämien um $157 \%$, die Leistungen dagegen um $169 \%$. Das wäre noch nicht so dramatisch. Betrachten wir aber die Bruttoschadenssätze von 10 Jahren, so lagen sie anfangs bei $66,8 \%$, im Jahre 1981 aber bei $72 \%$. Die Problematik wird noch deutlicher, wenn wir die Schadenversicherung ohne die Sparten Unfall und Kranken betrachten. Wir haben von 1972 bis 1981 eine Prämiensteigerung von $142 \%$ und gleichzeitig eine Steigerung der Leistungen von $153 \%$. Der Schadenssatz lag im Durchschnitt der 10 Jahre bei 67,3\% und im Jahre 1981 bei $73,3 \%$. Nun gehört zur Beurteilung der Technik auch die Position Kosten. Wenn wir davon ausgehen, daß die österreichischen Versicherer etwa $30 \%$ Kostensatz ausweisen, dann haben wir in der Unfall/Schadenversicherung 1981 einen Schadenkostensatz (kombiniert) von $102 \%$, jedoch ohne Unfall und Kranken einen Satz von 103,3\%. 
Dies ist zwar nur ein Trend, aber er zeigt, daß wir mit Verlust, mit einem erheblichen technischen Verlust, arbeiten. Es gibt genügend Indizien dafür, daß die Ergebnisse des Jahres 1981 nicht zufallsbedingt schlechter sind als die früheren. Als Beispiel darf ich eine Sparte herausgreifen, um ihren Verfall in den letzten Jahren bzw. Jahrzehnten vor Augen zu führen. Gemeint ist Industrie-Feuer und Feuer-BU. Nach den Verbandsstatistiken betrug der durchschnittliche Prämiensatz in Promille der Versicherungssumme im Jahre 1975 in der Feuerversicherung noch 1,45. 1976 blieb er fast unverändert, sank aber in den Folgejahren auf 1,$32 ; 1,29 ; 1,27$ und im Jahr 1980 auf 1,21. Für das Jahr 1981 wird ein Satz von 1,18 Promille geschätzt. Dies bedeutet gegenüber dem Jahr 1975 einen Abfall um rund $19 \%$. Bei der Feuer-BU fiel der durchschnittliche Prämiensatz in Promille noch stärker, von 3,11 im Jahre 1975 auf 2,30 im Jahr 1981. Das ist ein Verfall um $35 \%$. Es ist kein Trost, daß man in der Bundesrepublik für das Jahr 1982 in der industriellen Feuerversicherung einen Satz von nur 1,05 und in der BU einen solchen von 2,2 prognostiziert. Die Rückversicherer haben in der BRD in drei Jahren über eine Milliarde DM, das heißt 7 Milliarden Schilling, Verluste gehabt. Allein die Münchener-Rück erlitt im letzten Geschäftsjahr (1981/1982) 248 Millionen Mark Gesamtverlust, davon 116 Millionen DM im Industriebereich. Ganz allgemein muß festgestellt werden, daß als Folge der schlechten technischen Ergebnisse auch die Kapitalbasis der Gesellschaften schlechter geworden ist.

In Österreich gelten die Solvabilitätsbestimmungen noch nicht. Es muß aber festgestellt werden, daß etliche Unternehmungen diesen Kapitalisierungsstandard, der in den Ländern der EG Vorschrift ist, nicht erreichen könnten. Vielmehr hat sich seit 1945 die Relation von Eigenkapital zum Prämienvolumen ständig verschlechtert. Dazu kommt noch, daß die österreichische Fiskalpolitik das Bestreben, den realen Wert des Eigenkapitals zu erhalten, steuerlich schwer bestraft. Ganz im Gegensatz zu Ländern wie die Schweiz, Großbritannien und skandinavischen Länder, wo es steuerlich möglich ist, Sicherheits-, Katastrophen- oder Großschädenrückstellungen ohne Erschwernisse zu dotieren. Diese Rücklagen haben zumindest teilweise Eigenkapitalcharakter und können dessen Garantiefunktion mittragen.

Nun einige positive Aspekte : Was hat die österreichische Versicherungswirtschaft in den letzten zwei Jahren getan, um dieser Strömung, die sich weltweit breit macht, gegenzusteuern ? Ich glaube, wir müssen auf beiden Seiten Maßnahmen treffen, nicht nur auf der Seite der Technik, sondern auch auf der Seite der Kosten. Wir haben im vergangenen Jahr - in der Wiener Zeitung ist diese Maßnahme am 22.5.1981 veröffentlicht worden - die Provisionen in der Kfz-Haftpflichtversicherung mit $7 \%$ der Nettoprämie bzw. einer Einmalprovision von $20 \%$ der ersten Jahresnettoprämie maximiert. Weniger darf es sein, mehr aber nicht. Wir haben hier auch strenge Kontrollen eingeführt und werden diese Kontrollen fortsetzen. Um zu verhindern, daß eventuell auf Nebensparten ausgewichen wird, haben wir uns im Verband entschlossen, auch hiefür eine Regelung einzuführen. Wir haben die Provisionen in der Teilkaskoversicherung mit $15 \%$, in der Vollkaskoversicherung mit $12,5 \%$, bezogen auf die vereinnahmte Prämie, maximiert. Dazu möchte ich sagen, daß in den eigenen Organisationen diese Sätze nicht gezahlt werden. Es gibt noch eine zweite Regelung: Wenn eine mindestens zweijährige Laufzeit vorgesehen ist, kann man Provisionen bis zu $30 \%$ in Teilkasko und bis $\mathrm{zu} 25 \%$ in Vollkasko aus der Jahresnettoprämie verguiten. Diese Maßnahme mußte schon deshalb eingeführt werden, weil sonst die Mehrleistungen 
an Provisionen nicht nur bei der Kfz-Haftpflicht, sondern auch in den Nebensparten eine Mehrbelastung des Konsumenten erwirkt hätten.

Wir haben auch in der industriellen Feuerversicherung Maßnahmen getroffen. Die Versicherungsgesellschaften haben Erklärungen abgegeben, daß sie sich grundsätzlich an den Tarif für Industrie und Gewerbe halten und nur die genehmigten Bedingungen und Klauseln anwenden werden. Auf die Tarifprämie wird in besonderen Fällen, insbesondere in komplizierten Konkurrenzfällen, ein Nachlaß von höchstens $20 \%$ gewährt. Ich weiß, diese Formulierung ist nicht ganz glücklich. Wir haben uns sehr lange damit befaßt, uns aber schließlich doch dazu entschlossen.

Desweiteren haben wir versucht, im Interesse unseres Images in der Lebensversicherung Sofortmaßnahmen zu ergreifen. Wie Sie wissen, kam es heuer im Sommer zu sehr unangenehmen Entwicklungen auf dem Markt. Die Konsumentenschützer haben uns massiv angegriffen, sie haben verschiedene Fälle aufgezeigt, wo Vermögensberater, Pseudomakler (diese Anschuldigung bezog sich sicher nicht auf die seriösen, anerkannten Makler oder Maklergesellschaften) Geschäfte gemacht haben, durch die Konsumenten geschädigt wurden. Wir haben im Verband einen Beschluß gefaßt, durch verschiedene Maßnahmen solche Mißstände auf dem Lebensversicherungsmarkt künftig $\mathrm{zu}$ verhindern.

Das Problem ist noch nicht ausgetragen, es wird sicherlich noch weitere Schwierigkeiten geben. Wir verfolgen diese Entwicklung und es wurde im Verband eine Stelle geschaffen, die von den Konsumenten direkt angesprochen werden kann. Wir werden noch weitere Maßnahmen diesbezüglich treffen.

Eine ganz wesentliche Zielsetzung für die Zukunft ist in unserer Bestrebung zu erkennen, für eine verbesserte Ausbildung unserer Mitarbeiter zu sorgen. So ist es uns gelungen, auf der Wirtschaftsuniversität Wien erstmals einen eigenen Hochschullehrgang einzurichten. Dabei handelt es sich um eine zweijährige Ausbildung. Das erste Jahr ist allgemeinen Aspekten der Betriebswirtschaftslehre gewidmet, im zweiten Jahr befaßt man sich mit Versicherungsbetriebsfragen und mit dem Management. Die Absolventen erhalten den Titel "Akademisch geprüfter Versicherungskaufmann".

Gerade diese Maßnahme halte ich für überaus wichtig, obwohl auf den ersten Blick kein direkter Zusammenhang mit der gesamten technischen Versicherungsproblematik zu bestehen scheint. Doch je besser Versicherungskaufleute ausgebildet sind, desto besser wird das Kundenservice sein und umso besser wird auch der Konsument bei uns beraten. Das wird auch in Zukunft im Mittelpunkt der Bemühungen der Versicherungsunternehmen stehen.

Wenn auch - sowohl international als auch in Österreich - die derzeitige Lage auf dem Versicherungs- und Rückversicherungsmarkt mit Recht besorgniserregend ist, so läßt sich doch mit Befriedigung feststellen, daß in vielen Bereichen ernsthafte Anstrengungen gemacht werden, wieder zu technisch und kaufmännisch vernünftigem Verhalten zurückzukehren.

Ich bin sicher, daß wir Versicherer in unseren Bemühungen um eine Normalisierung des Marktgeschehens mit Ihrer tatkräftigen Unterstützung rechnen dürfen. 


\section{The Future Development of the Insurance Market - Summary *}

The continuous decrease of technical profits in nearly every country proves that also the insurance business has been affected by the world-wide crisis - contrary to a widely held belief that we in Austria are living on an isolated paradise.

The future development could be illustrated by five theses:

Thesis 1 : Since a number of years we are faced with an enormous overcapacity, not only in insurance but particularly so in reinsurance. Part of that capacity may be called "uninformed" or some times even more aptly "ignorant". This applies especially to those companies whose main objective is to penetrate into established markets at all cost. Misjudging the risks, they offer rates and conditions at which in the long run neither claims nor costs can be covered.

Thesis 2: Disregarding the inflation, taking into account simply the gross net produced by interests, lead to dangerous and erroneous conclusions. Would the rate of inflation been taken into proper consideration, it would soon become evident that the non-technical results are not at all realistic. In order to arrive at the right results, inflation rates should be substracted from the gross profits by interests. Nominal interests - in other words - are nothing but fictitious profits.

Thesis 3 : The so-called "cash-flow-underwriting" describes a company's policy whose main objective is to write as much business as possible - disregarding results - aiming to accrue more money for investment purposes.

In most cases this policy leads to an irresponsible underwriting, caring only for a high rate of interests hoping to achieve a "bottom-line-profit". This attitude pushes aside the well-known principle that first of all a technical profit has to be produced. Non-technical profits are there to counteract the inflation and to strengthen the reserves. Even, though, in many countries the reserves are highly taxed.

Thesis 4: Technical losses are not only produced by excessive competition, but sometimes are also caused by government interference.

Thesis 5: The correct interpretation of these symptoms should entice modern insurance managements to revert to the classical requirement of actuarial science, namely : An insurance company should dispose of adequate capital and reserves to enable it to cope with the ever changing risk situations and to be adequately strengthened to handle emergency cases if and when unforeseeable catastrophes occur.

This is the reason why the EEC applies such severe solvability rules regarding capital and reserves of insurance companies.

* By Karl Kornis, Chairman of the Board of Management, Erste Allgemeine, Vienna. 